\title{
Molecular breeding for resilience in maize - A review
}

\author{
Asima Gazal*1, Z. A. Dar ${ }^{2}$, A. A. Lone ${ }^{2}$, I. Abidi ${ }^{1}$ and G. Ali ${ }^{1}$ \\ ${ }^{1}$ Division of Genetics and Plant Breeding, Sher-e-Kashmir University of Agricultural Sciences and Technology of \\ Kashmir, Shalimar-190025 (J\&K), INDIA \\ ${ }^{2}$ Dryland (Karewa) Agricultural Research Station, Budgam- 190001 (J\&K), INDIA \\ *Corresponding author. E-mail: asimagazal@gmail.com
}

Received: March 21, 2015; Revised received: August 18, 2015; Accepted: September 26, 2015

\begin{abstract}
Abiotic and biotic constraints have widespread yield reducing effects on maize and should receive high priority for maize breeding research. Molecular Breeding offers opportunities for plant breeders to develop cultivars with resilience to such diseases with precision and in less time duration. The term molecular breeding is used to describe several modern breeding strategies, including marker-assisted selection, marker-assisted backcrossing, marker-assisted recurrent selection and genomic selection. Recent advances in maize breeding research have made it possible to identify and map precisely many genes associated with DNA markers which include genes governing resistance to biotic stresses and genes responsible for tolerance to abiotic stresses. Marker assisted selection (MAS) allows monitoring the presence, absence of these genes in breeding populations whereas marker assisted backcross breeding effectively integrates major genes or quantitative trait loci (QTL) with large effect into widely grown adapted varieties. For complex traits where multiple QTLs control the expression, marker assisted recurrent selection (MARS) and genomic selection (GS) are employed to increase precision and to reduce cost of phenotyping and time duration. The biparental mapping populations used in QTL studies in MAS do not readily translate to breeding applications and the statistical methods used to identify target loci and implement MAS have been inadequate for improving polygenic traits controlled by many loci of small effect. Application of GS to breeding populations using high marker densities is emerging as a solution to both of these deficiencies. Hence, molecular breeding approaches offers ample opportunities for developing stress resilient and high-yielding maize cultivars.
\end{abstract}

Keywords: Abiotic, Biotic, Maize, Molecular breeding, Stresses

\section{INTRODUCTION}

Plant development and production is affected by several abiotic and biotic stresses all across the world (Wani et al. 2013; Pathak et al., 2014). Biotic and abiotic stresses that have widespread yield-reducing effects and should receive high priority for maize breeding research include breeding for downy mildews, banded leaf and sheath blight, turcicum leaf blight, post-flowering stalk rot, stem borers, weevils and drought, water logging and acid soils (Gerpacio and Pingali 2007). Molecular breeding (MB) is the generic term used to describe several modern breeding strategies, including marker assisted selection (MAS), marker assisted backcrossing (MABC), marker assisted recurrent selection (MARS), and genomic selection (GS) (Ribaut et al., 2010). Marker assisted Selection (MAS) is the selection of specific alleles for traits conditioned by a few loci; Marker assisted backcrossing (MABC) is the transfer of a limited number of loci from one genetic background to another, including transgenes. Marker assisted recurrent selection (MARS) is the identification and selection of several genomic regions involved in the expression of complex traits to 'assemble the best performing genotype within a single, or across related, populations.
Genomic selection (GS) is selection based on markers without significance testing and without identifying a priori a subset of markers associated with the trait (Bernardo and $\mathrm{Yu}, 2007)$. Except GS which is still at the exploratory stage for plants, all these approaches are widely and successfully used in the private sector (Cooper et al., 2006, Crosbie et al., 2006; Eathington et al., 2007) but less so in the public sector, though there is some limited use in advanced institutions (Dwivedi et al., 2007; Ragot and Lee, 2007). Molecular breeding has led to development of plants resilient to various biotic (Roswarne et al. 2012, 2013, Yang et al. 2013) as well as abiotic stresses (Gosal et al., 2009). The potential applications of MB in crop plants for development disease resilience have been well discussed by Collard and Mackill 2008; Gupta et al., 2009; Prasanna et al. 2010b, Ibitoye and Akin-Idowu 2010 and $\mathrm{Xu}$ et al. 2013. Identification of several genes and quantitative trait loci (QTL) associated with abiotic and biotic stress tolerance in plants and their mapping has provided an abundance of DNA marker trait associations (Collard and Mackill 2008) and they not only assisted conventional breeders to develop stress tolerant cultivars in less time but also enable scientists to clone and characterize such genes / QTLs 
for developing genetically modified stress tolerant plants (Kumar et al. 2013). This review discusses about the molecular breeding strategies and recent developments and few successful examples related to development of stress tolerance in maize.

\section{Molecular breeding strategies}

Marker assisted selection: The use of DNA markers in plant breeding is called marker-assisted selection (MAS) and is a component of the new discipline of 'molecular breeding'. There are five main considerations for the use of DNA markers in MAS viz., reliability; quantity and quality of DNA required; technical procedure for marker assay; level of polymorphism and cost (Collard and Mackill, 2008). The last years have witnessed a continuous evolution of new molecular marker systems from morphological, biochemical and DNA-hybridization based molecular markers like restriction fragment length polymorphisms, random amplified polymorphic DNAs, and amplified fragment length polymorphisms to present-day popular marker systems such as simple sequence repeats (SSRs), single nucleotide polymorphisms (SNPs), and diversity array technologies (Dart). Advent of low-cost and high throughput sequencing technologies, commonly called next-generation sequencing (NGS) technologies have increased the speed of SSR and SNP discovery. NGS technologies in combination with restriction enzymes are now ready for detecting genome-wide polymorphism and new marker systems like RAD-tag sequencing, genotyping by sequencing are becoming popular. It seems that NGS-based marker systems will be dominating marker systems in future. These new emerging marker systems are expected to facilitate enhanced adoption of modern genetics and breeding approaches like genome-wide association studies and genomewide selection that generally require markers at highdensity in crop plants (Mir and Varshney, 2012).

Marker assisted backcross breeding (MABB): Backcrossing is a plant breeding method most commonly used to incorporate one or a few genes into an adapted or elite variety. In most cases, the parent used for backcrossing has a large number of desirable attributes but is deficient in only a few characteristics (Allard, 1999). The method was first described in 1922 and was widely used between the 1930s and 1960s (Stoskopf et al., 1993). The use of DNA markers in backcrossing i.e., marker-assisted backcrossing (MAB) can be described (Holland, 2004) in three levels, firstly the 'foreground selection' were markers are used in combination with or to replace screening for the target gene or QTL (Hospital and Charcosset, 1997). The second level 'recombinant selection' involves selecting backcross progeny with the target gene and recombination events between the target loci and linked flanking markers. This reduces the size of the introgression and 'linkage drag' (Hospital, 2005). Using conventional breeding methods, the donor segment can remain very large even with many $\mathrm{BC}$ generations (e.g. more than
10; Ribaut and Hoisington 1998; Salina et al., 2003). By using markers that flank a target gene (e.g. less than $5 \mathrm{cM}$ on either side), linkage drag can be minimized. Since double recombination events occurring on both sides of a target locus are extremely rare, recombinant selection is usually performed using at least two BC generations (Frisch et al., 1999). The third level called as 'background selection' involves selecting backcross progenies with the greatest proportion of recurrent parent genome, using markers that are unlinked to the target locus (Hospital and Charcosset 1997; Frisch et al., 1999) to accelerate the recovery of recurrent parent genome. Background markers are markers that are not linked to the target gene / QTL on all other chromosomes, in other words, markers that can be used to select against the donor genome.

Marker Assisted Backcross Breeding (MABB) Advantages:

MABB can allow selection for all kinds of traits to be carried out at seedling stage and it is faster and more accurate.

MABB is not influenced by $\mathrm{G} \times \mathrm{E}$, thus allowing the selection to be performed under any environmental conditions (e.g. greenhouse and off-season nurseries).

MABB using co-dominant markers (e.g. SSR and SNP) can allow effective selection of heterozygous individuals.

The presence of multiple genes governing a particular trait can be un-ambigously established.

Marker assisted gene pyramiding (MAGP): Marker Assisted Gene Pyramiding means assembling desirable genes from multiple parents into a single genotype for a specific trait. Marker assisted gene pyramiding has following advantages (Collard and Mackill, 2008).

Enhancing trait performance by combining two or more complementary genes.

Rectifying deficits by introgression of genes from other sources.

Increasing the durability of disease resistance.

For overcoming the limitations of MAS, MABB, particularly when multiple QTLs control the expression of complex traits new approaches like Marker Assisted Recurrent Selection (MARS) and Genomic Selection (GS) are used (Bernardo, 2010).

Marker assisted recurrent selection (MARS): Phenotypic recurrent selection involves cycles of selection, evaluation and recombination that aims at increasing the frequency of favorable allele within the population. If the same is based on markers it is called as marker assisted recurrent selection (MARS). In MARS, markers associated with trait of interest are first identified and selection is based on several genomic regions involved in the expression of complex traits to assemble the most superior genotype within a population (Ribaut et al., 2010). MARS is a scheme which allows performing genotypic selection and intercrossing in the same crop season for one cycle of selection. Therefore, MARS enhances the efficiency of 
phenotypic recurrent selection (Fig.1) and accelerates the progress of the procedure particularly helps in integrating multiple favorable genes/QTLs from different sources through recurrent selection based on a multipleparental population.

MARS involves estimation of marker effects from genotyping $\mathrm{F}_{2}$ or $\mathrm{F}_{3}$ population and phenotyping $\mathrm{F}_{2}$ derived $\mathrm{F}_{4}$ or $\mathrm{F}_{5}$ progenies, followed by two or three recombination cycles based on presence of marker alleles for small effect QTLs (Eathington et al., 2007). In the first step of MARS, de novo QTL identification is carried out initially, i.e. QTLs are identified in the breeding population itself, generally derived from good x good crosses. Subsequently, the lines carrying superior alleles for maximum QTLs are crossed to pyramid superior alleles in one genetic background. Recombined lines are then subjected to a final phenotypic screening to select the best lines for multilocation field testing to release them as varieties. MARS is particularly useful for capturing the several genomic regions especially to target more number of minor as well as major QTLs. Therefore, genetic gain achieved is higher by MARS as compared to the MABC program (Bernardo and Charcosset, 2006).

Genomic selection (GS): Genomic selection (GS) is a form of marker-based selection, which was defined by Meuwissen (2007) as the simultaneous selection for many (tens or hundreds of thousands of) markers, which cover the entire genome in a dense manner so that all genes are expected to be in linkage disequilibrium with at least some of the markers. In GS genotypic data (genetic markers) across the whole genome are used to predict complex traits with accuracy sufficient to allow selection on that prediction alone. Selection of desirable individuals is based on genomic estimated breeding value (GEBV) (Nakaya and Isobe, 2012), which is a predicted breeding value calculated using an innovative method based on genome-wide dense DNA markers (Meuwissen et al., 2001). A flow diagram of a genomic selection breeding program is described in figure-2. GS does not need significant testing and identifying a subset of markers associated with the trait (Meuwissen et al., 2001). In other words, QTL mapping with populations derived from specific crosses can be avoided in GS. However, it does first need to develop GS models, i.e. the formulae for GEBV prediction (Nakaya and Isobe, 2012). In this process (training phase), phenotypes and genome-wide genotypes are investigated in the training population (a subset of a population) to predict significant relationships between phenotypes and genotypes using statistical approaches. Subsequently, GEBVs are used for the selection of desirable individuals in the breeding phase, instead of the genotypes of markers used in traditional MAS. For accuracy of GEBV and GS, genome-wide genotype data is necessary and require high marker density in which all quantitative trait loci (QTLs) are in linkage disequilibrium with at least one marker.
GS in plants was less studied and large-scale empirical studies are not available in public sectors for plant breeding (Jannink et al., 2010), but it has attracted more and more attention in recent years (Bernardo, 2010; Bernardo and Yu, 2007; Guo et al., 2011; Heffner et al., 2010, 2011; Lorenzana and Bernardo, 2009; Wong and Bernardo, 2008; Zhong et al., 2009). In plants, GS was first demonstrated for Maize, $A$. thaliana and Barley by Lorenzana and Bernardo (2009). Studies indicated that in all cases, accuracies provided by GS were greater than might be achieved on the basis of pedigree information alone (Jannink et al., 2010). Also, in plants, the importance of generation time varies between crops, but the goal of reducing cycle time remains. In maize, a crop that uses doubled haploids and off season nurseries, test cross performance selection still requires at least two years (Bernardo and $\mathrm{Yu}, 2007$ ), providing an opportunity for GS to reduce unit time per selection cycle by reducing the need for progeny test data in every cycle.

Molecular breeding for biotic stresses in maize: Various biotic and abiotic stresses have widespread yield-reducing effects on maize (Gerpacio and Pingali 2007). QTLs that have been utilized in resilience breeding programmes are discussed below with few examples from turcicum leaf blight, Polysora rust and drought tolerance.

Downy mildew resistance: High priorities are given to downy mildew resistance development and several QTLs governing downy mildew resistance have been identified and significant one mapped on chromosome 6 using a RIL set developed from crosses between Ki3 (resistant) and CML139 (George, Prasanna, and Rathore 2003). In the Asian Maize Biotechnology Network (AMBIONET) study, 135 RIL families were evaluated for downy mildew reactions (during year 2000 and 2001) at different locations, viz., Mandya (southern India) against sorghum downy mildew $(P$. sorghi); at Farm Suwan (Thailand) against sorghum downy mildew (P. zeae); at Maros (Indonesia) against Java downy mildew (P. maydis); at Udaipur (western India) against Rajasthan downy mildew ( $P$. heteropogoni); and at Southern Mindanao (Philippines) against Philippine downy mildew (P. philippinensis). AMBIONET study identified three SSR markers viz., umc11, $u m c 23 a$, and umc113 linked tightly to the QTL on chromosome 6, suggesting their possible use for MAS (Prasanna and Hoisington 2003). In India, QTL each on chromosome 3 and 6 were identified and validated from backcross mapping population developed by a cross between NAI116 (Sorghum downy mildew resistant) and CM139 (Nair, Prasanna, and Garg 2005). Marker assisted backcross breeding generated sorghum downy mildew resistant line (CM139) and significant number of QTL-NILs which would be further used to develop resilient maize cultivars (Prasanna 2009a). Identification and mapping of major QTLs helps in developing DNA marker trait associations (Collard and Mackill 2008) which help in developing resilience in 


\section{Recurrent Selection Scheme}

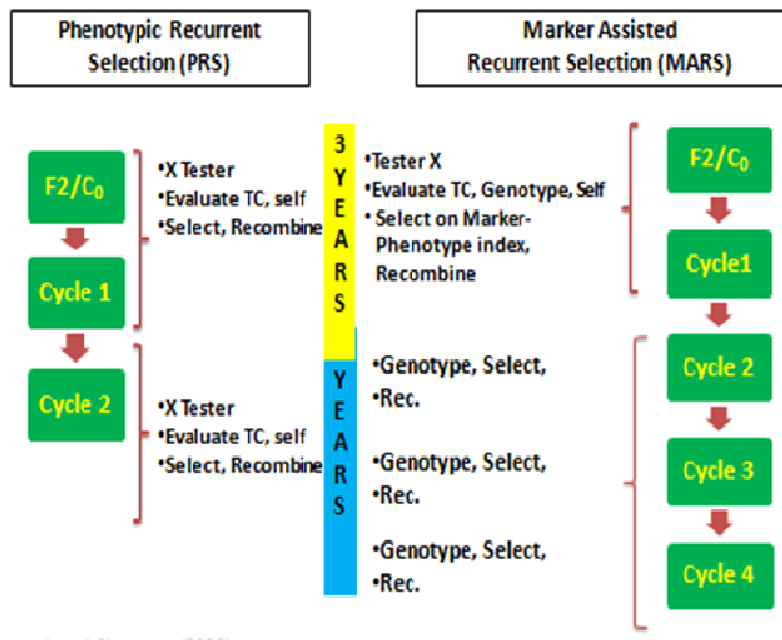

Fig. 1. Recurrent Selection Scheme (comparison between Phenoypic recurrent selection and marker assisted recurrentselection), (Source: Bernardo, R., and A. Charcosset. 2006. Usefulness of gene information in marker-assisted recurrent selection: a simulation appraisal. Crop Sci. 46: 614-21).

susceptible cultivars and also chances for successful application of MAS for resistance development are better when QTLs are identified in the germplasm.

Turcicum leaf blight and polysora rust: Molecular marker assisted pyramiding of major genes governing resistance to turcicum leaf blight and Polysora rust in elite five Indian lines viz., CM137, CM138, CM139, CM140 and CM212 has been achieved at Indian Agricultural Research Institute (Prasanna et al. 2010a; Prasanna et al. 2009b). Turcicum leaf blight resistant genes i.e., Htnland $H t 2$ along with a QTL (RppQ) for Polysora rust from four resistant donors viz., NAI 147, SKV 21, NAI 112 and SKV18 were pyramided together by generating seven different backcross populations.

Northern corn leaf blight resistance (NCLB): Studies on resistance to NCLB point to a complex genetic nature with many quantitative trait loci distributed genome wide (Van Inghelandt et al. 2012; Poland et al. 2011). Genomic selection in maize for prediction of northern corn leaf blight resistance was employed by Technow, Burger, and Melchinger 2013 by using training sets combining both heterotic groups thereby increasing prediction accuracies. Genomic BLUP model was used to predict genotypic values of 100 dent and 97 flint lines which were genotyped with high -density SNP marker and phenotyped for NCLB resistance per se.

Banded leaf and sheath blight (BLSB) resistance: The BLSB disease, caused by Rhizoctonia solani Kuhn in maize, is one of the most destructive and important diseases of maize in South and Southeast Asia. Very few sources of resistance to this disease have been found. In China, a mapping population consisting of 229 F2 individuals derived from the cross of inbreds

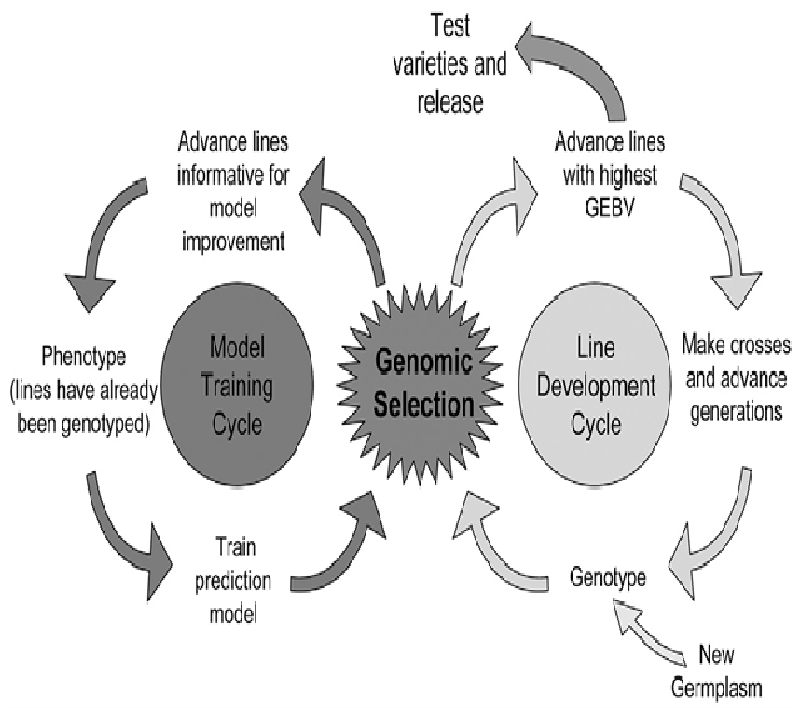

Fig. 2. Flow diagram of a genomic selection breeding program. Breeding cycle time is shortened by removing phenotypic evaluation of lines before selection as parents for the next cycle. Model training and line development cycle length will be crop and breeding program specific. $(G E B V=g e-$ nomic estimated breeding value) (Heffner et al., 2009).

R15 (resistant) and 478 (susceptible) were used to map QTLs conferring resistance to BLSB. Four QTLs out the eleven significant QTLs for resistance (located on chromosomes 2, 6 and 10) were stable across locations, accounting for $3.72-10.35 \%$ of the phenotypic variation (Zhao et al. 2006a, b). In another study in India, a F2:3 mapping population was generated using CA00106 (BLSB-tolerant) and CM140 (BLSBsusceptible). Phenotyping was undertaken using artificial BLSB inoculation at three locations (Delhi, Pantnagar and Udaipur) which are 'hot spots' for the disease. QTL mapping revealed location-specific QTLs for BLSB resistance, with most of the favorable QTL alleles contributed by the resistant parent CA00106. The study also led to identification of three QTLs (on chr. 6, 8 and 9) with significant epistatic interactions (Garg et al., 2009). The studies on BLSB in Asia have so far revealed a high degree of genotype $\mathrm{x}$ environment interaction, and complex nature of inheritance of resistance to the disease. It is important to intensify efforts to identify stable and additional sources of resistance to BLSB and improve the disease resistance of present maize hybrids.

Molecular breeding for abiotic stresses in maize drought tolerance: Breeding drought tolerant maize is the most focused research worldwide as maize is highly sensitive to drought mainly during reproductive stages. Marker assisted backcross breeding was used to incorporate several QTL alleles for short interval between anthesis and silking (ASI period) from Ac7643 (drought-tolerant) donor to CML247 (Ribaut and Ragot 2007). A major QTL, Root-ABA1 identified by Giuliani et al. 2005 is related to root development along with abscisic acid levels in leaf under water 
stress and with stomatal conductance. Recent trends and advances in molecular breeding in India and China (Prasanna et al. 2009a; Hao, Li, and Xie 2008; Xiao, $\mathrm{Li}$ and George 2005) have led to identification of QTLs associated with tolerance to drought tolerance on different chromosomes (Prasanna et al. 2009a). QTLs identified from recombinant inbred lines (RILs) on chromosome 1 , 2, 8 and 10 were found to influence specific traits under drought stress. Also, a digenic epistatic QTL for kernel number per ear under drought stress was identified (Prasanna et al. 2010b). Analysis of a mapping population $\left(\mathrm{F}_{2: 3}\right)$ derived from the cross between drought tolerant line X178 and B73 [at the Chinese Academy of Agricultural Sciences (CAAS)] (Xiao, Li and George 2005; Hao, $\mathrm{Li}$ and Xie 2008) at different locations in central and southern China resulted in detection of a major QTL for ASI (anthesis silking interval) and ear number per plant under drought stress on chromsome 1 (bin 1.03) and chromsome 9 (bins 9.03-9.05), which correspond to some major QTLs identified in different experiments on drought stress worldwide (Tuberosa, Salvi and Giuliani 2007). Such 'consensus QTLs' identified in maize for drought tolerance would be utilized in marker-assisted breeding programmes as good candidates to improve maize production and productivity under drought conditions (Prasanna et al. 2010b). Almeida et al. 2013 evaluated three tropical bi-parental (CML444 x MALAWI; CML440 x CML504; CML444 x CML441) populations under well-watered \& water-stress treatments in Kenya, Mexico, and Zimbabwe to identify genomic regions responsible for grain yield (GY) and anthesis-silking interval (ASI). Meta-QTL (mQTL) analysis identified one genomic region for ASI and seven regions for GY. Among these six mQTL were expressed on chromosomes $1,4,5$ and 10 for GY were across treatments and environments. One mQTL on chr.7 for GY and one on chr.3 for ASI were found to be 'adaptive' under stress conditions. These mQTL regions can be effectively used in the MAS and MARS programs for developing drought tolerance.

Excess soil moisture (ESM) tolerance: Excess Soil Moisture affects over 18 per cent of the total maize production area in South and Southeast Asia, causing production losses of 25 to 30 per cent annually (Zaidi et al. 2010). In India, water logging is the second most serious constraint for crop production after drought. Significant QTLs have been identified for waterlogging tolerance at seedling stage (Qiu et al. 2007). Mano et al. (2005) identified QTLs on chromosomes 3, 7, and 8, for adventitious root formation under excess soil moisture condition from $\mathrm{F}_{2}$ population of a cross between a maize inbred line, B64 and teosinte (Zea mays ssp. Huehuetenangensis). Similarly, Mano et al. (2009) identified QTLs controlling constitutive aerenchyma formation under flooding conditions on chromosomes 1, 5, and 8 from cross between another teosinte accession (Zea mays spp. Nicaraguensis) and maize inbred line B73. The production of NILs with such QTLs in maize would be beneficial for improvement of tolerance towards excess soil moisture. QTL analysis to map the genes controlling adventitious root formation on the soil surface (ARF-SS) under flooding conditions were undertaken in the seedlings of $317 \mathrm{BC}_{3} \mathrm{~F}_{1}$ progenies derived from a cross between elite maize Mi29 and teosinte Zea nicaraguensis (Mano et al. 2009). From interval mapping analysis and single point regression the QTLs for ARF-SS were detected on chromosomes 3 (bin 3.04), 7 (bin 7.04) and 8 (bin 8.03) (Mano et al. 2009). Six QTL (ph6-1, sdw4-1, sdw7-1, tdw4-1, tdw7-1 and rll-2,) were identified at seedling stage associated with plant height, shoot dry weight, total dry weight root length and root dry weight, detected at three stages viz., the period during 0 to 3 days, 3 to 6 days and the period during 6 to 9 days of waterlogging by Osman et al. 2013.

\section{Conclusion}

Molecular Breeding offers opportunities for plant breeders to develop cultivars with resilience to diseases with precision and in less time duration as discussed above. MB approaches like MAS, MABB, MARS and GS have provided various new avenues and opportunities for plant breeders which are quite evident from the adoption and success of these approaches in many cases in almost every crop. MB is an efficient approach to increase genetic gain per crop cycle. Hence, efforts of plant breeders, molecular biologists and scientists in meeting the food requirements on a sustainable basis for ever increasing population are not hampered. Molecular breeding and conventional breeding are complementary in most breeding programmes as there are various issues and bottlenecks which hinder MB strategies especially in developing countries like high cost, non availability and complexity of molecular platforms, reliability of marker profiling and scoring, limited markers and degree of polymorphism, gene / QTL x E effects, lack of equipment, resources, technical expertise and lack of application gap. Thus, to meet these challenges in molecular breeding, platforms (policies) need to be developed to reduce cost and to optimize MARS and GWS procedures to identify high-yielding, resilient and stable genotypes (with low $\mathrm{G} \times \mathrm{E} \times \mathrm{M}$ ). The emergence of public-private partnership platforms for accessing MB tools with support services and ever increasing demand for improved disease resilient varieties to counter the food crisis throughout the globe predict that MB strategies will have a significant impact on crop improvement breeding programmes prevailing in developing countries.

\section{REFERENCES}

Allard, R.W. (1999). Principles of plant breeding, 2nd edn. New York, NY: Wiley.

Bernardo, R. (2010). Genome wide selection with minimal crossing in self-pollinated crops. Crop Sci., 50: 624627.

Bernardo, R. and Charcosset, A. (2006). Usefulness of gene information in marker-assisted recurrent selection: a simulation appraisal. Crop Sci., 46: 614-21.

Bernardo, R. and Yu, J. (2007). Prospects for genome wide selection for quantitative traits in maize. Crop Sci., 47: 1082-1090. 
Collard, B.C.Y. and Mackill, D.J. (2008). Marker-assisted selection: an approach for precision plant breeding in the twenty-first century. Philos. Trans. R. Soc. Lond. B Biol. Sci., 363: 557-572.

Cooper, M., Smith, O.S., Merrill, R.E., Arthur, L., Polich, D.W. and Loffler, C.M. (2006). Integrating breeding tools to generate information for efficient breeding: past, present, and future. In Plant Breeding: The Arnel R. Hallauer International Symposium, 141-154.

Crosbie, T.M., Eathington, S.R., Johnson, G.R., Edwards, M., Reiter, R., Stark, S., Mohanty, R.G., Oyervides, M., Buehler, R.E. and Walker, A.K. (2006). Plant breeding: past, present, and future. In Plant Breeding: The Arnel $R$. Hallauer International Symposium, 3-50.

Dwivedi, S.L., Crouch, J.H., Mackill, D.J., Xu, Y., Blair, M.W., Ragot, M.,Upadhyaya, H.D. and Ortiz, R. (2007). The molecularization of public sector crop breeding: progress, problems, and prospects. Advances in Agronomy, 7: 3-8.

Eathington, S.R., Crosbie, T.M., Edwards, M.D., Reiter, R.S. and Bull, J.K. (2007). Molecular markers in commercial breeding. Crop Science, 47: 154-163.

Frisch, M., Bohn, M. and Melchinger, A.E. (1999). Minimum sample size and optimal positioning of flanking markers in marker-assisted backcrossing for transfer of a target gene. Crop Sci., 39: 967-975.

Garg, A., Prasanna, B.M. and Sharma, R.C. (2009). Genetic analysis and mapping of QTLs for resistance to banded leaf and sheath blight (Rhizoctonia solani f.sp. sasakii) in maize. In: Proceedings of 10th Asian regional maize workshop (October 20-23, 2008, Makassar, Indonesia). CIMMYT, Mexico DF (in press).

George, M.L.C, Prasanna, B.M. and Rathore, R.S. (2003). Identification of QTLs conferring resistance to downy mildews of maize in Asia. Theor. Appl. Genet., 107: 544-551.

Gerpacio, R.V. and Pingali, P.L. (2007). Tropical and subtropical maize in Asia: production systems, constraints, and research priorities. CIMMYT, Mexico DF.

Goddard, M.E. and Hayes, B.J. (2007). Genomic selection. $J$. Anim. Breed. Genet.124: 323-330.

Gosal, S.S., Wani, S.H. and Kang, M.S. (2009). Biotechnology and drought tolerance. Journal of Crop Improvement 23: 19-54.

Guo, Z., Tucker, D.M., Liu, J., Kishore, V. and Gay, G. 2011. Evaluation of genome wide selection efficiency in maize nested association mapping populations. Theor. Appl. Genet., 124: 261-275.

Gupta, P.K., Langridge, Peter and Mir, R.R. (2009). Markerassisted wheat breeding: present status and future possibilities. Mol. Breeding 26(2): 145-161

Hao, Z., Li, X. and Xie, C. (2008). Two consensus quantitative trait loci clusters controlling anthesis-silking interval, ear setting and grain yield might be related with drought tolerance in maize. Ann. Appl. Biol., 153: 7383.

Heffner, E.L., Jannink, J.L., Iwata, H., Souza, E. and Sorrells, M.E. (2011). Genomic selection accuracy for grain quality traits in biparental wheat populations. Crop Sci., 51: 2597-2606.

Heffner, E.L., Lorenz, A.J., Jannink, J.L. and Sorrells, M.E. (2010). Plant breeding with genomic selection: gain per unit time and cost. Crop Sci., 50: 1681-1690.

Heffner, E.L., Sorrells, M.E. and Jannink, J.L. (2009). Genomic selection for crop improvement. Crop Sc., 49: 1-
12.

Holland, J. B. (2004) Implementation of molecular markers for quantitative traits in breeding programs-challenges and opportunities. In Proc. 4th Int. Crop Sci. Congress. Brisbane, Australia, 26 September-1 October.

Hospital, F. (2005). Selection in backcross programmes. Phil. Trans. R. Soc. B., 360: 1503-1511.

Hospital, F. and Charcosset, A. (1997). Marker-assisted introgression of quantitative trait loci. Genetics, 147: 1469-1485.

Ibitoye, D.O. and Akin-Idowu, P.E. (2010). Marker-assistedselection (MAS): A fast track to increase genetic gain in horticultural crop breeding. African Journal of Biotechnology 9 (52): 8889-8895.

Jannink, J.L., Lorenz, A.J. and Iwata, H. (2010). Genomic selection in plant breeding: from theory to practice. Briefings in Functional Genomics, 9: 166-177.

Kumar, S., Kumari, P., Kumar, U., Grover, M., Singh, A.K., Singh, R. and Sengar, R.S. (2013). Molecular approaches for designing heat tolerant wheat. J. Plant Biochem. Biotechnol. DOI 10.1007/s13562-013-0229-3.

Lorenzana, R. E. and Bernardo, R. (2009). Accuracy of genotypic value predictions for marker-based selection in biparental plant populations. Theor. Appl. Genet., 120: 151-161.

Mano, Y., Omori, F., Loaisiga, C. H., and Bird, R. M. (2009). QTL mapping of aboveground adventitious roots during flooding in maize $\mathrm{x}$ teosinte "Zea nicaraguensis" backcross population. Plant Root, 3: 3-9.

Mano, Y., Omori, F., Muraki, M., and Takamizo, T. (2005). QTL mapping of adventitious root formation under flooding conditions in tropical maize. Breed. Sci., 55: 343-347.

Meuwissen, T. (2007). Genomic selection: marker assisted selection on a genome wide scale. J. Anim. Breed. Genet., 124: 321-322.

Meuwissen, T.H.E., Hayes, B.J. and Goddard, M.E. (2001). Prediction of total genetic value using genome wide dense marker maps. Genetics, 157: 1819-1829.

Mir, R.R. and Varshney, R.K. (2012). Future Prospects of Molecular Markers in Plants. Molecular Markers in Plants. John Wiley \& Sons, Inc.

Nair, S.K., Prasanna, B.M., Garg, A. (2005). Identification and validation of QTLs conferring resistance to sorghum downy mildew (Peronosclerospora sorghi) and Rajasthan downy mildew ( $P$. heteropogoni) in maize. Theor. Appl. Genet., 110:1384-1392.

Nakaya, A. and Isobe, S.N. (2012). Will genomic selection be a practical method for plant breeding? Annals of Botany, DOI:10.1093/aob/mcs109.

Osman, K.A., Tang, B., Wang, Y., Chen, J., Yu, F., Li, L., Han, X., Zhang, Z., Yan, J., Zheng, Y., Yue, B., and Qiu, F. (2013). Dynamic QTL Analysis and Candidate Gene Mapping for Waterlogging Tolerance at Maize Seedling Stage. PLOS ONE 8(11): e79305. doi:10.1371/ journal.pone.0079305.

Pathak, M.R., Teixeira da Silva, J.A. and Wani, S.H. (2014). Polyamines in response to abiotic stress tolerance through transgenic approaches. GM Crops 5(1): 1-10.

Poland, J.A., Bradbury, P.J., Buckler, E.S. and Nelson, R.J. (2011). Genome-wide nested association mapping of quantitative resistance to northern leaf blight in maize. Proc. Natl. Acad. Sci. USA. 108(17): 6893-6898.

Prasanna, B.M., Beiki, A.H., Sekhar, J.C., Srinivas, A. and Ribaut, J.M. (2009a). Mapping QTLs for component 
traits influencing drought stress tolerance of maize in India. J. Plant Biochem. Biotech. 18:151-160.

Prasanna, B.M., Hettiarachchi, K. and Mahatman, K. (2009b). Molecular marker-assisted pyramiding of genes conferring resistance to Turcicum leaf blight and Polysora rust in maize inbred lines in India. In: Proceedings of $10^{\text {th }}$ Asian regional maize workshop (October 20 -23, 2008, Makassar, Indonesia). CIMMYT, Mexico DF (in press).

Prasanna, B.M., Mahatman, K.H., Rajan, A., Singh, O.N., Kaur, B., Zaidi, P.H., Azrai, M. and Pixley, K.N. (2010a). Molecular marker-assisted pyramiding of genes conferring resistance to Turcicum leaf blight and Polysora rust in maize inbred lines in India. In: Proceedings of $10^{\text {th }}$ Asian regional maize workshop (October 20-23, 2008, Makassar, Indonesia). CIMMYT, Mexico DF.

Prasanna, Pixley, B.M. K., Warburton, M.L. and Xie, C.X. (2010b). Molecular marker-assisted breeding options for maize improvement in Asia. Mol. Breeding 26: 339-356.

Qiu, F., Zheng, Y., Zhang, Z., and Xu, S. (2007). Mapping of QTL associated with water-logging tolerance during the seedling stage in maize. Ann. Bot., 99: 1067-1081.

Ragot, M. and Lee, M. (2007). Marker-assisted selection in maize: current status, potential, limitations and perspectives from the private and public sectors. In Markerassisted Selection: Current Status and Future Perspectives in Crops Livestock Forestry and Fish. Rome: Food and Agriculture Organization of the United Nations: 117-150.

Ribaut, J.M. and Hoisington, D. (1998). Marker-assisted selection: new tools and strategies. Trends Plant Sci., 3: 236-239.

Ribaut, J.M. and Ragot, M. (2007). Marker-assisted selection to improve drought adaptation in maize: The backcross approach, perspectives, limitations, and alternatives. $J$. Exp. Bot. 58: 351-360.

Ribaut, J.M., Jiang, C. and Hoisington, D. (2002). Simulation experiments on efficiencies of gene introgression by backcrossing. Crop Sci., 42: 557-565.

Ribaut, J.M., Vicente, M.C. and Delannay, X. (2010). Molecular breeding in developing countries: challenges and perspectives. Current Opinion in Plant Biology, 13: 1-6.

Rosewarne, G.M., Herrera-Foessel, S.A., Singh, R.P., Huerta -Espino, J., Lan, C.X. and He, Z.H. 2013. Quantitative trait loci of stripe rust resistance in wheat. Theor. Appl. Genet.126: 2427-2449.

Rosewarne, G.M., Singh, R.P., Huerta-Espino, J., HerreraFoessel, S.A., Forrest, K.L., Hayden, M.J. and Rebetzke, G.J. (2012). Analysis of leaf and stripe rust severities reveals pathotype changes and multiple minor QTLs associated with resistance in an Avocet $\times$ Pastor wheat population. Theor. Appl. Genet. 124: 1283-1294.

Salina, E., Dobrovolskaya, O., Efremova, T., Leonova, I. and Roder, M.S. (2003). Microsatellite monitoring of re- combination around the Vrn-B1 locus of wheat during early backcross breeding. Plant Breed., 122: 116-119.

Stoskopf, N.C., Tomes, D.T. and Christie, B.R. (1993). Plant breeding: theory and practice. San Francisco, CA; Oxford: Westview Press Inc.

Technow, F., Burgerand, A. and Melchinger, A.E. (2013). Genomic Prediction of Northern Corn Leaf Blight Resistance in Maize with Combined or Separated Training Sets for Heterotic Groups. G3: Genes Genomics Genetics 3: 197-203.

Tuberosa, R., Salvi, S. and Giuliani, S. (2007). Genome-wide approaches to investigate and improve maize response to drought. Crop Sci., 47: S120-S141.

Van Inghelandt, D., Melchinger, A.E., Martinan, J.P. and Stich, B. (2012). Genome-wide association mapping of flowering time and northern corn leaf blight (Setosphaeria turcica) resistance in a vast commercial maize germplasm set. BMC Plant Biol. 12: 56.

Wani, S.H., Singh, N.B., Haribhushan, A. and Mir, J.I. (2013). Compatile solute engineering in plants for abiotic stress tolerance- role of glycine betaine. Current genomics 14(3): 157.

Wong, C.K. and Bernardo, R. (2008). Genomewide selection in oil palm: increasing selection gain per unit time and cost with small populations. Theoretical and Applied Genetics, 116(6): 815-824.

Xiao, Y.N., Li, X.H. and George, M.L. (2005). Quantitative trait loci analysis of drought tolerance and yield in maize in China. Plant Mol. Biol. Reporter, 23:155-165.

Xu, Y., Xie, C., Wan, J., He, Z. and Prasanna, B.M. 2013. Marker-Assisted Selection in Cereals: Platforms, Strategies and Examples. P. K. Gupta and R. K. Varshney (eds.), Cereal Genomics II (C) Springer DOI: 10.1007/978-94-007-6401-9-14.

Yang, E.N., Rosewarne, G.M., Herrera-Foessel, S.A., Huerta -Espino, J., Tang, Z.X., Sun, C.F., Ren, Z.L. and Singh, R.P. (2013). QTL analysis of the spring wheat "Chapio" identifies stable stripe rust resistance despite intercontinental genotype $\times$ environment interactions. Theor. Appl. Genet. 126: 1721-1732.

Zaidi, P. H., Maniselvan, P., Srivastava, A., Yadav, P., and Singh, R. P. (2010). Genetic analysis of water-logging tolerance in tropical maize (Zea mays L.). Maydica, 55: 17-26.

Zhao, M., Zhang, Z. and Zhang, S. (2006a). Quantitative trait loci for resistance to Banded Leaf and Sheath Blight in maize. Crop Sci., 46:1039-1045.

Zhao, W., Canaran, P. and Jurkuta, R. (2006b). Panzea: a database and resource for molecular and functional diversity in the maize genome. Nucl. Acids Res., 34: D725-D757.

Zhong, S., Dekkers, J.C.M., Fernando, R.L. and Jannink, J.L. (2009). Factors affecting accuracy from genomic selection in populations derived from multiple inbred lines: a barley case study. Genetics, 182: 355-364. 